\title{
ASP5094, a humanized monoclonal antibody against integrin alpha-9, did not show efficacy in patients with rheumatoid arthritis refractory to methotrexate: results from a phase $2 \mathrm{a}$, randomized, double-blind, placebo-controlled trial
}

Tsutomu Takeuchi ${ }^{{ }^{*}}$ D, Yoshiya Tanaka ${ }^{2}$, Jay Erdman ${ }^{3}$, Yuichiro Kaneko ${ }^{4}$, Masako Saito ${ }^{4}$, Chieri Higashitani ${ }^{4}$, Ronald Smulders ${ }^{3}$ and Christopher Lademacher ${ }^{3}$

\begin{abstract}
Background: Rheumatoid arthritis (RA) is a chronic, debilitating autoimmune condition characterized by joint synovial inflammation. Current treatments include methotrexate (MTX), biologic agents, and Janus kinase (JAK) inhibitors. However, these agents are not efficacious in all patients and there are concerns regarding side effects and risk of infection as these treatments target immune-related pathways. Overexpression and activation of integrin alpha-9 (a9) on fibroblast-like synoviocytes are associated with RA disease onset and exacerbation. The humanized immunoglobulin G1 monoclonal antibody ASP5094 was designed to inhibit human a9 and is currently under investigation for the treatment of RA.

Methods: This phase 2a, multicenter, randomized, placebo-controlled, double-blind, parallel-group study (NCT03257852) evaluated the efficacy, safety, and biological activity of intravenous ASP5094 $10 \mathrm{mg} / \mathrm{kg}$ in patients with moderate to severe RA that was refractory to MTX. Patients received ASP5094 or placebo every 4 weeks for a total of three administrations. Both treatment groups used concomitant MTX. The primary efficacy endpoint was the proportion of patients who responded per American College of Rheumatology 50\% improvement using C-reactive protein (ACR50-CRP) after 12 weeks of treatment. Biological activity of ASP5094 was assessed via pharmacokinetics and pharmacodynamics of known downstream effectors of a9. Safety was also assessed.

(Continued on next page)
\end{abstract}

\footnotetext{
* Correspondence: tsutake@z5.keio.jp

${ }^{1}$ Keio University School of Medicine, 35 Shinanomachi, Shinjuku City, Tokyo 160-8582, Japan

Full list of author information is available at the end of the article
}

C C The Author(s). 2020 Open Access This article is licensed under a Creative Commons Attribution 4.0 International License, which permits use, sharing, adaptation, distribution and reproduction in any medium or format, as long as you give appropriate credit to the original author(s) and the source, provide a link to the Creative Commons licence, and indicate if changes were made. The images or other third party material in this article are included in the article's Creative Commons licence, unless indicated otherwise in a credit line to the material. If material is not included in the article's Creative Commons licence and your intended use is not permitted by statutory regulation or exceeds the permitted use, you will need to obtain permission directly from the copyright holder. To view a copy of this licence, visit http://creativecommons.org/licenses/by/4.0/. The Creative Commons Public Domain Dedication waiver (http://creativecommons.org/publicdomain/zero/1.0/) applies to the data made available in this article, unless otherwise stated in a credit line to the data. 
(Continued from previous page)

Results: Sixty-six patients were enrolled and randomized to placebo $(n=33)$ or ASP5094 ( $n=33)$. In the primary efficacy analysis, ACR50-CRP response rates were 6.3\% and 18.2\% at week 12 in the ASP5094 and placebo groups, respectively; a difference of -11.9 , which was not significant (2-sided $P$ value $=0.258$ ). No trends in ACR50 response rates were observed in subgroups based on demographics or baseline disease characteristics, and no significant differences between placebo and ASP5094 were identified in secondary efficacy or pharmacodynamic endpoints, despite achievement of target serum concentrations of ASP5094. Most treatment-emergent adverse events were mild to moderate in severity, and ASP5094 was considered safe and well tolerated overall.

Conclusion: Although no notable safety signals were observed in this study, ASP5094 was not efficacious in patients with moderate to severe RA with an inadequate response to MTX.

Trial registration: ClinicalTrials.gov, NCT03257852. Registered on 22 Aug. 2017

Keywords: Antibodies, Monoclonal, Humanized, Antirheumatic agents, Arthritis, Rheumatoid, Biological products, Extracellular matrix proteins, Integrins, Integrin alpha-9, human, Methotrexate, Synovial membrane, Synoviocytes

\section{Introduction}

Rheumatoid arthritis (RA) is a chronic, systemic, inflammatory, autoimmune disease characterized by joint synovial inflammation. This condition is associated with irreversible cartilage destruction and osteolysis, resulting in pain, disability, interference in activities of daily living, and reduced quality of life [1-3]. To control synovitis and subsequent irreversible joint damage, the recommended course of treatment consists of diseasemodifying antirheumatic drugs (DMARDs), initiated as soon as possible upon diagnosis of RA, with dosages adjusted to treat to a target of clinical remission, or if that is not reached, to a target of low disease activity [4].

Methotrexate (MTX) is a first-line therapy for the treatment of RA, and when the target is not achieved, biologic agents or Janus kinase (JAK) inhibitors can be added [5,6]. Even though biologic DMARDs have several mechanisms of action, including inhibiting the activity of cytokines such as tumor necrosis factor-alpha (TNF- $\alpha$ ) and interleukin (IL)-6 [7, 8], T-cell costimulatory pathways, and CD20 on B cells $[9,10]$, only about one half to two thirds of patients achieve clinical remission [11, 12], and these treatments are associated with the risk of infectious events [13-16]. In addition, molecular signatures characteristic of RA do not achieve normal levels following treatments targeting TNF or IL6 , even if the patient achieves clinical remission [17]. Thus, the need remains for safer and more effective treatments for RA, potentially through targeting novel mechanisms of action.

The disease process of RA involves activated fibroblast-like synoviocytes (FLS) in affected joints. These cells can lead to cartilage and bone degradation through production of matrix metalloproteinases (MMPs), upregulation of receptor activator of nuclear factor $\mathrm{kB}$ ligand (RANKL), production of IL-6, and recruitment and activation of proinflammatory immune cells [18-22]. Recent findings suggest distinct types of
FLS may be important for chronic inflammation and bone erosion in RA and may activate lymphocytes via antigen presentation, and when present in the joint lining, be a major cause of joint destruction [23-25].

Integrin family proteins are composed of two subunits, $\alpha$ and $\beta$, and they bind to extracellular matrix (ECM) components and regulate a wide range of cellular responses such as migration, survival, and proliferation [26]. Integrin alpha-9 $(\alpha 9)$ binds to ECM proteins such as tenascin-C [27], protease-cleaved osteopontin [28], and VCAM-1 [29]; contributes to cell adhesion and migration [30, 31]; and is highly expressed in synovial tissue cells, especially FLS, in patients with RA [32, 33]. This protein is also expressed on FLS of arthritic joints [34] and is overexpressed prior to onset of arthritis in mouse models [35]. Monoclonal antibodies against $\alpha 9$ alleviated mouse collagen-induced arthritis (CIA) [34, 36] and significantly reduced FLS-derived biomarkers [37]. Interestingly, treatment of CIA with the $\alpha 9$ antibody neither altered spleen cell numbers nor decreased plasma levels of anti-type II collagen antibody. Additionally, $\alpha 9$ antibody did not induce a mixed lymphocyte reaction or delayed type hypersensitivity reaction [37]. Thus, $\alpha 9$ appears to be a promising target for new therapeutics in the treatment of RA with minimal suppression of protective immunity.

ASP5094 is a humanized immunoglobulin G1 monoclonal antibody that targets human $\alpha 9$. Phase 1 data from healthy adult volunteers and patients with RA showed ASP5094 to be safe and well tolerated [38]. The present study investigated the efficacy, safety, pharmacokinetics (PK), and pharmacodynamics (PD) in patients with RA refractory to MTX treatment.

\section{Patients and methods \\ Study design}

This was a phase $2 \mathrm{a}$, multicenter, randomized, placebocontrolled, double-blind, parallel-group study conducted 
at 31 centers in Japan to evaluate the efficacy, safety, and PK of ASP5094 $(10 \mathrm{mg} / \mathrm{kg})$ in patients with moderate to severe active RA despite the use of MTX (Clinicaltrials. gov identifier: NCT03257852). Patients were randomized 1:1 to ASP5094 or placebo groups at baseline after a 28day screening period, then received treatment with study drug every 4 weeks for a total of three administrations (day 1, week 4, and week 8; Fig. 1). Patients also received concomitant MTX (oral formulation) within the approved dose range. This study was performed in compliance with good clinical practice.

\section{Study patients}

Eligible patients were male or female, aged 20 years or older, had a diagnosis of RA based on the 1987 American College of Rheumatology (ACR) criteria or the 2010 ACR/European League Against Rheumatism (EULAR) criteria for at least 6 months prior to screening, and met 1991 ACR Revised Criteria for the Classification of Global Functional Status in RA Class I, II, or, III. Criteria for active RA included at least six tender joints (based on 68-joint assessment), at least six swollen joints (based on 66-joint assessment), and C-reactive protein (CRP) levels exceeding $0.50 \mathrm{mg} / \mathrm{dL}$. Eligible patients were also required to have received MTX continuously for at least 90 days prior to screening and be able to continue a stable dose of MTX from $\geq 28$ days prior to screening through the study period. Patients were excluded from the study if they had ongoing infection that required antibiotics, had inflammatory arthritis other than RA (e.g., psoriatic arthritis, ankylosing spondylitis, systemic lupus erythematosus, sarcoidosis, gouty arthritis), had other articular symptoms that may have affected joint assessment, or showed evidence of autoimmune disease other than RA (excluding Sjogren's syndrome and chronic thyroiditis), history of or concurrent malignant tumor, or other severe, progressive, or uncontrolled illness.
Individuals were also excluded if they had previously shown an inadequate response to biologic DMARDs or JAK inhibitors; this exclusion criterion was implemented because the response rates of those in the refractory patient population could be lower, and targeting a more homogeneous population was a reasonable way to detect an efficacy signal in this small proof-of-concept study. Written informed consent was obtained from all patients prior to any study-related procedures.

\section{Study treatment}

ASP5094 was prepared at the study site by unblinded staff by adding test drug to saline to be administered at a dose of $10 \mathrm{mg} / \mathrm{kg}$ in a total volume of approximately $100 \mathrm{~mL}$. Placebo consisted of a matching solution of approximately $100-\mathrm{mL}$ saline prepared to be indistinguishable from the active treatment. During treatment visits study patients received the prepared ASP5094 $10 \mathrm{mg} / \mathrm{kg}$ solution or placebo via intravenous (IV) infusion for approximately $30 \mathrm{~min}$.

\section{Efficacy endpoints}

The primary efficacy endpoint was the response rate (i.e., proportion of patients) according to ACR 50\% improvement criteria assessed using the CRP level (ACR50-CRP) at week 12 . ACR50 response rates at weeks $1,2,4,8$, and 16 , and ACR20/70 at the same time points, plus week 12 assessments were determined as secondary endpoints. Additional secondary endpoints included change from baseline in the Disease Activity Score (DAS) 28-CRP or DAS28-erythrocyte sedimentation rate (ESR) score, Simplified Disease Activity Index (SDAI) score, and Clinical Disease Activity Index (CDAI) score through week 16, as well as rates of remission and low disease activity based on DAS28 score $(<2.6$ and $\leq 3.2$, respectively), "good" or "moderate" response according to EULAR criteria, and remission based on ACR/EULAR score.

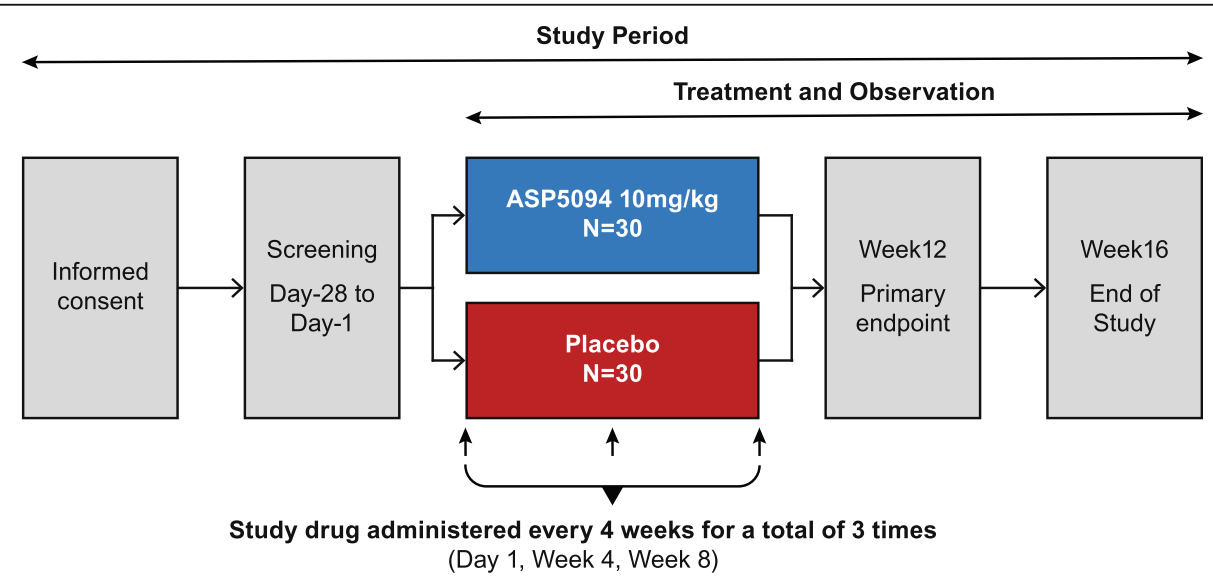

Fig. 1 Study design 


\section{Pharmacokinetics}

Blood samples were collected at baseline and at each scheduled visit to determine serum concentrations of ASP5094. Samples were collected once each during the visits on week 1 , week 2 , week 12/discontinuation, and week 16/discontinuation, then 4 weeks later; and twice (before and after study drug administration) on day 1 (baseline), week 4, and week 8. The blood samples taken after study drug administration were generally collected within $15 \mathrm{~min}$ after the end of treatment from the patient's arm opposite from where study drug was administered. Serum samples were diluted to 1:100 in caseincontaining sample buffer and incubated on a microtiter plate, MULTI-ARRAY 96-Well Plate (Standard, Meso Scale Diagnostics, LLC), coated with an antibody to capture ASP5094, then were washed three times with PBS containing $0.05 \%$ Tween 20 and incubated with a luminescent detection antibody that was quantified and analyzed by electrochemiluminescence immunoassay (ELCI A), using SECTOR (Meso Scale Discovery, Rockville, MD). ASP5094-specific capture, as well as biotin-labeled secondary antibodies, were purposefully created for this assay and are not commercially available.

\section{Pharmacodynamics}

Pharmacodynamic assays were performed to assess the activity of ASP5094 on the known downstream effectors of $\alpha 9$ in RA of TNF- $\alpha$, MMP-3, and IL- 6 , which were measured at baseline and each scheduled visit. These serum concentrations were measured by commercially available kits as follows: TNF- $\alpha$; ELISA, Quantikine HS ELISA (R\&D Systems), MMP-3; LTIA, Panaclear MMP3: Latex (Sekisui Medical), IL-6; CLEIA, IL-6 LPG Immunoreaction Cartridges (Fujirebio). Levels of putative $\alpha 9$ ligands tenascin (TNC)-C (large variant of tenascin$\mathrm{C}$, termed FNIII-B variant), vascular cell adhesion molecule (VCAM)-1, and osteopontin (OPN; full-length and thrombin-cleaved) were determined at baseline and week 12/discontinuation and week 16/discontinuation, then 4 weeks later for exploratory evaluation of ASP5094 on potential biomarkers. Plasma concentrations of TNCC, VCAM-1, and OPN were measured by a commercially available ELISA kit as follows: TNC-C; Tn-C large [FNIII-B] assay kit (Immuno-Biological Laboratories), VCAM-1; Human sVCAM1/CD106 Quantikine ELISA kit; (R\&D Systems), OPN full-length; Human osteopontin Quantikine ELISA kit (R\&D Systems), cleaved-OPN; Human osteopontin N-half assay kit (Immuno-Biological Laboratories).

\section{Safety}

Safety was assessed via incidence of treatment-emergent adverse events (TEAEs) over the duration of the study, and by electrocardiogram, vital signs, bodyweight and laboratory assessments comprising hematology, biochemistry, and urinalysis at each scheduled visit.

\section{Sample size determination and statistical analyses}

Based on findings of previous clinical studies in RA [11, 39-41], and assuming ACR50 response rates at week 12 would be $10 \%$ in the placebo group and $50 \%$ in the ASP5094 group, a sample size of 26 patients per group was expected to provide $90 \%$ power to detect a difference between the groups at a 2 -sided significance level of 0.10 . Therefore, 60 patients were planned to be randomized in a $1: 1$ ratio to ASP5094 $(n=30)$ or placebo $(n=30)$.

\section{Statistical analyses}

Efficacy analyses were based on the full analysis set (FAS), defined as all patients who received at least one dose of study drug and had at least one efficacy measurement after study drug administration. The safety analysis set (SAF) used for safety analyses consists of all patients who took at least one dose of study drug. The PK and PD analyses were based on corresponding analysis sets that comprised patients who received study drug and from whom at least one PK and PD analysis sample, respectively, was collected after administration.

For comparisons of binary variables, Fisher's exact test was used with a significance level of 10\% (2-sided), unless otherwise indicated. Differences of response rates between treatment groups and 2-sided 90\% confidence interval (based on the normal approximation) were also calculated. Continuous variables were compared using an analysis of covariance, with treatment group as a factor and baseline score as a covariate; mean and standard deviation (SD) of actual values and changes from baseline were displayed. For missing data, nonresponder imputation (NRI) was used for the primary analysis and last observation carried forward (LOCF) for all secondary endpoints.

\section{Results \\ Patients}

Among 91 recruited patients, 66 were enrolled and randomized to placebo $(n=33)$ or ASP5094 $(n=33)$ and comprised the SAF. A total of 59 patients (89.4\%) completed the study; 1 (3.0\%) and six (18.2\%) patients from the placebo and ASP5094 groups, respectively, discontinued prior to study end (Fig. 2). The FAS included 65 patients (placebo, $n=33$; ASP5094, $n=32$ ) as one patient in the ASP5094 group was excluded from the analyses for having no data for the efficacy endpoints.

The mean age of randomized patients was 56.3 years and 47 (70.8\%) were female. Demographic characteristics and baseline disease activity were similar across treatment groups (Table 1). 


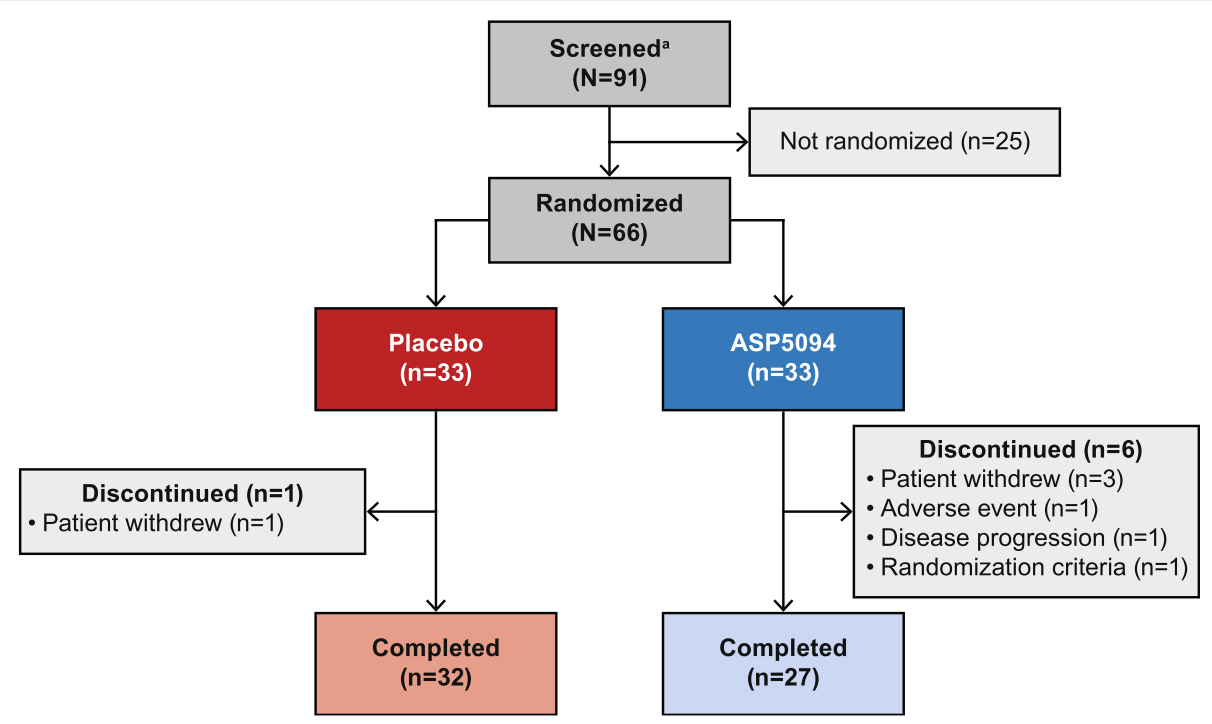

Fig. 2 Patient disposition. ${ }^{\text {PProvided informed consent }}$

\section{Efficacy}

For the primary efficacy endpoint of ACR50-CRP response rates at week 12, the proportion of patients in ASP5094 group $(2 / 32 ; 6.3 \%)$ was not higher than in the placebo group $(6 / 33 ; 18.2 \%)$. This represented a difference of $-11.9 \%$ between groups, which was not statistically significant $(P=0.258$; Table 2$)$. Rates of ACR50CRP response over time are provided in Supplemental Fig. 1. Subgroup analyses were performed to determine whether particular demographic or disease characteristics, such as age, sex, MTX dose, duration of RA, and baseline disease activity, may affect treatment outcomes. Subgroup analysis did not reveal any obvious trends in ACR50 response rate between ASP5094 and placebo (Supplemental Table 1).

Secondary ACR efficacy endpoints at week 12 included ACR20 and ACR70 response rates. Similar to the results observed with ACR50, the proportion of patients in the ASP5094 group who achieved these endpoints was not higher than in the placebo group (Table 2).

When additional secondary endpoints were examined, findings failed to demonstrate significant improvement in ASP5094 across all timepoints. Specifically, the change from baseline in DAS28-CRP (Fig. 3a), DAS28ESR (Fig. 3b), SDAI (Fig. 3c), and CDAI (Fig. 3d) values demonstrate that ASP5094 did not lead to statistically significant or clinically meaningful improvements, compared with placebo.

\section{Pharmacokinetics/pharmacodynamics}

A summary of serum concentrations of ASP5094 following $10 \mathrm{mg} / \mathrm{kg}$ dose administration on day 1 and at week 4 and week 8 is presented in Table 3. The peak mean serum ASP5094 concentration was achieved at the end of infusion. A trend of increasing mean serum ASP5094 concentrations prior to dosing was observed at each successive treatment visit, suggesting a steady state of ASP5094 serum concentration was not achieved after three doses.

The mean change and percent changes from baseline in TNF- $\alpha$, MMP-3, IL-6, TNC-C, VCAM-1, and OPN (full-length and thrombin-cleaved) at week 12 are summarized in Supplemental Table 2. The change from baseline in PD endpoints generally showed large variability, and there were no obvious differences in any of these endpoints between the ASP5094 and placebo groups.

\section{Safety}

The incidence of TEAEs was $39.4 \%$ and $60.6 \%$ in the placebo and ASP5094 groups, respectively (Table 4). The only TEAEs with an incidence $\geq 10 \%$ in any treatment group were worsening of RA (placebo: $n=4$ [12.1\%]; ASP5094: $n=6$ [18.2\%]) and viral upper respiratory tract infection (placebo: $n=3$ [9.1\%]; ASP5094: $n=$ 4 [12.1\%]). Most TEAEs were mild to moderate in severity, except for severe events of bronchitis and influenza in one patient in the ASP5094 group. The incidence of drug-related TEAEs was $6.1 \%$ and $15.2 \%$ in the placebo and ASP5094 groups, respectively. One patient in the placebo group experienced a serious TEAE (spinal compression fracture) while two patients in the ASP5094 group experienced serious TEAEs (severe bronchitis and influenza considered to be possibly drug related in one patient, and two events of tendon rupture in the other patient who used a steroid). The events of tendon rupture were considered attributable to joint deformation caused by joint destruction and daily motion and were assessed to be not related to study drug by the physician. 
Table 1 Patient demographics, disease history, and baseline disease characteristics

\begin{tabular}{|c|c|c|}
\hline \multicolumn{3}{|l|}{ Parameter } \\
\hline Demographics and disease history $^{a}$ & $\begin{array}{l}\text { Placebo } \\
(n=33)\end{array}$ & $\begin{array}{l}\text { ASP5094 } \\
(n=33)\end{array}$ \\
\hline Age, years, mean $\pm S D$ & $57.9 \pm 9.1$ & $55.7 \pm 13.8$ \\
\hline Median (range) & $57.0(41-77)$ & $55.5(30-77)$ \\
\hline$\geq 65$ years, $n(\%)$ & $9(27.3 \%)$ & $10(30.3 \%)$ \\
\hline Female, $n(\%)$ & $26(78.8 \%)$ & $21(63.6 \%)$ \\
\hline Weight, $\mathrm{kg}$, mean $\pm \mathrm{SD}$ & $57.82 \pm 11.13$ & $62.83 \pm 15.16$ \\
\hline $\mathrm{BMl}, \mathrm{kg} / \mathrm{m}^{2}$, mean $\pm \mathrm{SD}$ & $22.81 \pm 4.09$ & $24.33 \pm 4.20$ \\
\hline Duration of RA, years, mean \pm SD & $9.28 \pm 8.27$ & $9.87 \pm 9.41$ \\
\hline $\begin{array}{l}\text { MTX dose at screening, mg/week, } \\
\text { mean } \pm \text { SD }\end{array}$ & $10.08 \pm 2.86$ & $9.65 \pm 3.46$ \\
\hline$>0 \leq 8 \mathrm{mg} /$ week, $n(\%)$ & $10(30.3)$ & $15(45.5)$ \\
\hline$>8 \leq 12$ mg/week, $n(\%)$ & $18(54.5)$ & $11(33.3)$ \\
\hline > 12 mg/week, n (\%) & $5(15.2)$ & $7(21.2)$ \\
\hline $\begin{array}{l}\text { Baseline disease activity, } \\
\text { mean } \pm S D^{b}\end{array}$ & $\begin{array}{l}\text { Placebo } \\
(n=33)\end{array}$ & $\begin{array}{l}\text { ASP5094 } \\
(n=32)\end{array}$ \\
\hline Tender joint count (68 joints) & $12.3 \pm 5.7$ & $12.6 \pm 5.1$ \\
\hline Swollen joint count (66 joints) & $11.2 \pm 5.1$ & $11.5 \pm 4.6$ \\
\hline $\begin{array}{l}\text { Patient's global assessment of } \\
\text { arthritis pain }^{a}\end{array}$ & $42.58 \pm 23.20$ & $54.42 \pm 23.13$ \\
\hline $\begin{array}{l}\text { Patient's global assessment of } \\
\text { arthritis }^{\text {a }}\end{array}$ & $47.94 \pm 22.36$ & $51.42 \pm 23.83$ \\
\hline $\begin{array}{l}\text { Physician's global assessment of } \\
\text { arthritis }^{\mathrm{a}}\end{array}$ & $51.82 \pm 16.20$ & $52.89 \pm 15.78$ \\
\hline $\mathrm{CRP}, \mathrm{mg} / \mathrm{dL}$ & $1.406 \pm 1.031$ & $1.668 \pm 1.418$ \\
\hline $\mathrm{ESR}, \mathrm{mm} / \mathrm{h}$ & $42.48 \pm 21.26$ & $43.09 \pm 24.55$ \\
\hline DAS28-CRP score & $4.98 \pm 0.68$ & $5.15 \pm 0.61$ \\
\hline DAS28-ESR score & $5.66 \pm 0.79$ & $5.67 \pm 0.82$ \\
\hline SDAl score & $28.90 \pm 8.39$ & $30.32 \pm 8.21$ \\
\hline CDAl score & $27.49 \pm 8.17$ & $28.65 \pm 8.10$ \\
\hline HAQ-DI score & $0.750 \pm 0.537$ & $0.754 \pm 0.712$ \\
\hline
\end{tabular}

${ }^{\mathrm{a}}$ Safety analysis set

${ }^{\mathrm{b}}$ Full analysis set

'Based on 100-mm analog scale

$B M I$ body mass index, CDAl Clinical Disease Activity Index, CRP C-reactive protein, DAS28 Disease Activity Score in 28 Joints, ESR erythrocyte sedimentation rate, FAS full analysis set, $H A Q-D /$ Health Assessment Questionnaire-Disability Index, MTX methotrexate, $R A$ rheumatoid arthritis, SD standard deviation, SDAl, Simplified Disease Activity Index

No deaths were reported during the study. No clinically significant laboratory abnormalities were detected in either group, and there were no remarkable differences in changes in laboratory values between groups.

\section{Discussion}

The development of biologic agents targeting cytokines, $\mathrm{T}$ cells, and $\mathrm{B}$ cells marked a revolutionary change in the treatment of RA. However, not all patients achieve treatment targets, and some experience adverse events such
Table 2 ACR response rates at week 12

\begin{tabular}{|c|c|c|}
\hline & Placebo $(n=33)$ & ASP5094 $(n=32)$ \\
\hline ACR50 responders, $n(\%)^{a}$ & $6(18.2)$ & $2(6.3)$ \\
\hline Difference $(90 \% \mathrm{Cl})$ & $-11.9(-25.0,1.2)$ & \\
\hline$P$ value $^{\mathrm{b}}$ & 0.258 & \\
\hline ACR20 responders, $n(\%)^{c}$ & $16(48.5)$ & $12(37.5)$ \\
\hline Difference $(90 \%$ Cl) & $-11.0(-31.1,9.1)$ & \\
\hline$P$ value ${ }^{b}$ & 0.455 & \\
\hline ACR70 responders, $n(\%)^{c}$ & $2(6.1)$ & $1(3.1)$ \\
\hline Difference $(90 \% \mathrm{Cl})$ & $-2.9(-11.4,5.6)$ & \\
\hline$P$ value ${ }^{b}$ & 1.000 & \\
\hline
\end{tabular}

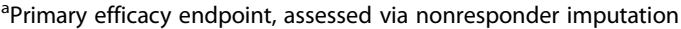

${ }^{\mathrm{b}} P$ values based on Fisher's exact test

'Secondary efficacy endpoint, assessed via last observation carried forward technique

ACR American College of Rheumatology, Cl confidence interval

as severe infections, highlighting the need for new therapeutic targets. Accumulating evidence supports activation and/or increased expression of $\alpha 9$ as a key mechanism in the RA disease process [20, 32, 42] including $\alpha 9$ overexpression on FLS in rheumatic joints preceding the onset of arthritis $[18,19,35]$, and the activation of $\alpha 9$ stimulating transformation of FLS into a pathologic state that includes hyperplastic and proinflammatory activity [33]. Moreover, monoclonal antibodies against $\alpha 9$ suppressed arthritis development and reduced FLS-derived biomarkers while sparing systemic immune activity in mice [37]. Thus, $\alpha 9$ was identified as a potential target for therapeutic development in RA.

In the current study, we investigated the clinical efficacy, safety, and PK and PD effects of the monoclonal $\alpha 9$ blocking antibody ASP5094 in patients with moderate to severe active RA. Our results revealed that $10 \mathrm{mg} / \mathrm{kg}$ ASP5094 was not more efficacious than placebo plus MTX at improving signs or symptoms of RA. The primary efficacy endpoint of ACR50 response rate at week 12 showed no clinically meaningful or statistically significant improvement with ASP5094 versus placebo, and no meaningful differences between treatment groups were observed in any secondary endpoints. Exploratory biomarkers were highly variable and there was no clear indication of $\mathrm{PD}$ modulation by ASP5094. We also did not observe any clear differences or trends between treatments within subgroups classified by demographics or potential stratification biomarkers, further indicating a lack of clinical efficacy by ASP5094.

The dose of $10 \mathrm{mg} / \mathrm{kg}$ tested in this study was chosen based on the findings in a previous phase 1 study [38, 43] in which binding of ASP5094 to $\alpha 9$ on neutrophils was assessed via percent receptor occupancy (\%RO). In that study, $3 \mathrm{mg} / \mathrm{kg}$ ASP5094 led to RO above $80 \%$ for 85 days, and above $90 \%$ for 141 days with $10 \mathrm{mg} / \mathrm{kg}$ ASP5094. RO was not assessed in the current study, yet 


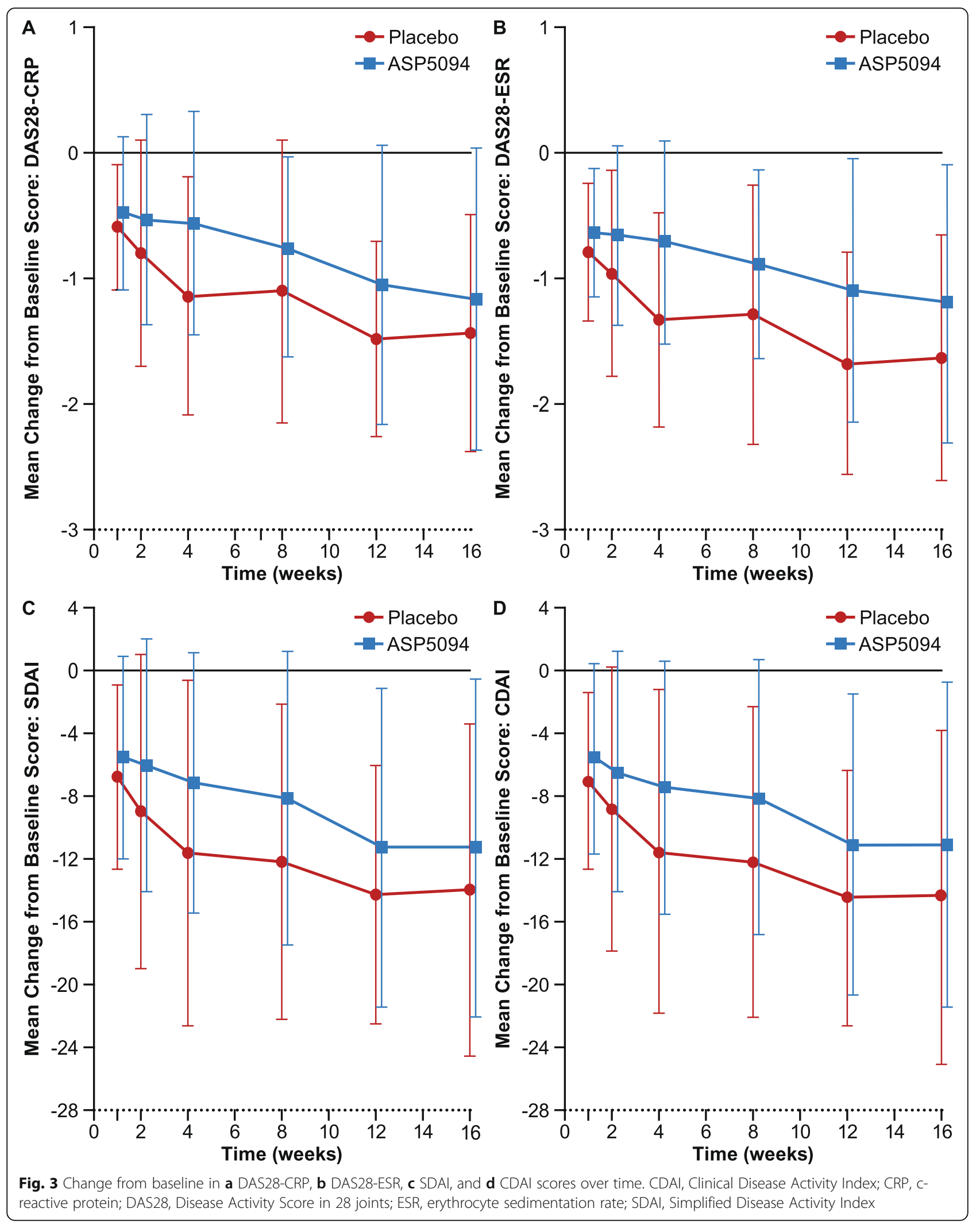




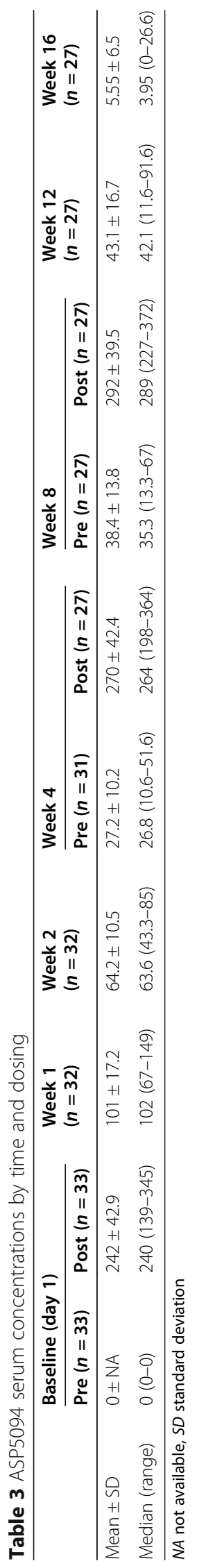


Table 4 Summary of TEAEs

\begin{tabular}{lll}
\hline $\boldsymbol{n}(\%)$ & Placebo $(\boldsymbol{n}=\mathbf{3 3})$ & ASP5094 ( $\mathbf{n}=\mathbf{3 3})$ \\
\hline TEAE & $13(39.4 \%)$ & $20(60.6 \%)$ \\
$\quad$ Mild & $9(27.3 \%)$ & $13(39.4 \%)$ \\
$\quad$ Moderate & $4(12.1 \%)$ & $6(18.2 \%)$ \\
$\quad$ Severe & 0 & $1(3.0 \%)$ \\
Drug-related TEAE & $2(6.1 \%)$ & $5(15.2 \%)$ \\
Serious TEAE & $1(3.0 \%)$ & $2(6.1 \%)$ \\
Drug-related serious TEAE & 0 & $1(3.0 \%)$ \\
TEAE leading to permanent discontinuation & 0 & $2(6.1 \%)$ \\
Drug-related TEAE leading to discontinuation & 0 & $1(3.0 \%)$ \\
Serious TEAE leading to permanent discontinuation & 0 & 0 \\
Death & 0 & 0 \\
TEAEs experienced by $>$ 1 patient overall & & $6(18.2 \%)$ \\
$\quad$ Rheumatoid arthritis & $4(12.1 \%)$ & $4(12.1 \%)$ \\
$\quad$ Viral upper respiratory tract infection & $3(9.1 \%)$ & $3(9.1 \%)$ \\
Constipation & $1(3.0 \%)$ & $2(6.1 \%)$ \\
Influenza & $1(3.0 \%)$ & $1(3.0 \%)$ \\
Chest discomfort & $2(6.1 \%)$ & $1(3.0 \%)$ \\
Cystitis & $1(3.0 \%)$ & $1(3.0 \%)$ \\
Pneumonia & $1(3.0 \%)$ & \\
\hline TEAE & & \\
\end{tabular}

TEAE treatment-emergent adverse event

minimum serum ASP5094 concentrations ranged from 10.6 to $13.3 \mu \mathrm{g} / \mathrm{mL}$ at the end of each dosing period and was $11.6 \mu \mathrm{g} / \mathrm{mL}$ at week 12 , which was more than 100 fold higher than the mean day 85 predose level $(3 \mathrm{mg} / \mathrm{kg}$ dose group) in the previous study. Therefore, the target serum ASP5094 level was likely achieved in the current study, and the lack of clinical effectiveness or PD effects occurred while serum ASP5094 concentrations were within the expected range.

There are several potential explanations for the lack of efficacy we observed. First, the favorable preclinical data for ASP5094 that supported clinical investigation was obtained using a mouse model of acute arthritis in which administration of anti-mouse $\alpha 9$ antibody suppressed type II collagen-induced arthritis in both prophylactic and therapeutic regimens [37]. In contrast with the mouse, increased interstitial pressure by hyperplasia and extracellular matrix deposition in humans with RA might prevent drug diffusion from blood vessels to synovial tissues. Although our PK results suggest adequate serum levels of ASP5094 were achieved, these measurements were performed in peripheral blood, which does not necessarily confirm that ASP5094 occupied and suppressed $\alpha 9$ in the target tissue. A discrepancy between circulating ASP5094 and target tissue ASP5094 could explain the lack of PD effects; however, these data are not feasible to obtain in patients. Second, extremely high local concentrations of multiple $\alpha 9$ ligands such as OPN, tenascin-C, or VCAM-1 may compete with ASP5094 for binding to $\alpha 9$ integrin. In our previous study, ASP5094 neutralized binding of $\alpha 9$ to its ligand with an IC50 value of around $1 \mathrm{ng} / \mathrm{mL}$, indicating that the antibody had good neutralizing potency in vitro ([33], Fig. 7A). However, in another study, a higher concentration of ASP5094 was required to suppress in vitro action of FLS derived from RA patients ([33], Fig. 7C). One possible explanation for this discrepancy is that huge amounts of ligands in the system produced by FLS affected the neutralizing activity of ASP5094. Additionally, there are data that suggest OPN levels in synovial fluid are markedly elevated compared with levels in the blood in patients with RA [43], and these may out-compete $\alpha 9$ for ASP5094 binding. Moreover, increased levels of locally expressed $\alpha 9$ might absorb a significant portion of circulating ASP5094, thereby depleting the amount available for binding in the synovium. Finally, the disease model in the aforementioned preclinical studies was of acute arthritis, which may not adequately represent the complex nature of chronic human RA. It is possible that the $\alpha 9$ neutralization that suppressed synovium activation and yielded positive results in the mouse model is not sufficient by itself to treat human RA where direct modulation of immune-pathway activation through additional targets may also be required. Thus, 
$\alpha 9$ alone may not be an effective target in the treatment of RA.

Although the incidence of overall and drug-related TEAEs was higher in the ASP5094 group, the overall incidence of severe and/or serious TEAEs was low. Overall, no notable safety signals were observed in this study, but further safety data will be needed in order to ascertain the overall safety profile of ASP5094 in patients with RA.

Single-cell analysis data from synovial tissue samples were published in 2019 by Zhang et al. [25]. Based on the information provided by these types of innovative technologies, it might be interesting to consider testing ASP5094 in patients specifically characterized by $\alpha 9$ overexpression.

\section{Conclusions}

In this study of patients with moderate to severe active RA refractory to MTX treatment, $10 \mathrm{mg} / \mathrm{kg}$ ASP5094 was not effective for the treatment of RA. Despite the lack of efficacy, no notable safety signals were observed in this study. Results of PK and PD assessments suggested that the expected serum levels of ASP5094 were achieved, yet expected molecular target effects were not observed. These findings may suggest target site exposure to ASP5094 in RA tissue was insufficient, or that targeting integrin $\alpha 9$ alone may not be adequate to deactivate FLS and thus improve RA.

\section{Supplementary information}

Supplementary information accompanies this paper at https://doi.org/10. 1186/s13075-020-02336-3.

Additional file 1: Supplemental Table 1. Analyses of ACR50-CRP response at Week 12 by baseline characteristic (NRI; FAS). Supplemental Table 2. Result, change, and percent change from baseline in pharmacodynamics at Week 12. Supplemental Fig. 1 ACR50-CRP response over time.

\section{Abbreviations}

a9: Integrin alpha-9; ACR: American College of Rheumatology; ACR50CRP: American College of Rheumatology 50\% improvement using C-reactive protein; BMI: Body mass index; CDAl: Clinical Disease Activity Index; CRP: Creactive protein; DMARDs: Disease-modifying antirheumatic drugs; ELISA: Enzyme-linked immunosorbent assay; ESR: Erythrocyte sedimentation rate; EULAR: European League Against Rheumatism; FAS: Full analysis set; FLS: Fibroblast-like synoviocytes; IV: Intravenous; JAK: Janus kinase; LOCF: Last observation carried forward; MMP: Matrix metalloproteinases; MTX: Methotrexate; NRI: Nonresponder imputation; OPN: Osteopontin; PD: Pharmacodynamic; PK: Pharmacokinetic; RA: Rheumatoid arthritis; RO: Receptor occupancy; SD: Standard deviation; SDAl: Simplified Disease Activity Index; TNC-C: Tenascin-C; TEAE: Treatment-emergent adverse event; VCAM: Vascular cell adhesion molecule

\section{Acknowledgements}

This study was funded by Astellas Pharma, Inc. Medical writing/editorial support was provided by Nate Connors, PhD; Laura L McCormick, PhD; and Elizabeth Hermans, PhD, from OPEN Health Medical Communications, Chicago, IL, and funded by the study sponsor.

\section{Authors' contributions}

$\Pi$, YT, JE, YK, CH, and $\mathrm{CL}$ designed the research study. $\mathrm{CH}$ performed the research. $\mathrm{CH}$ acquired the data. $\mathrm{YK}$ and $\mathrm{CL}$ analyzed the data. $\Pi, \mathrm{YT}, \mathrm{JE}, \mathrm{YK}$, $M S, C H, R S$, and $C L$ interpreted the data. TT, YT, JE, YK, MS, CH, RS, and CL critically reviewed and revised manuscript. All authors approved the final version of the manuscript.

\section{Funding}

This study was funded by Astellas Pharma, Inc.

\section{Availability of data and materials}

Researchers may request access to anonymized participant level data, trial level data, and protocols from Astellas sponsored clinical trials at www. clinicalstudydatarequest.com. For the Astellas criteria on data sharing see https://clinicalstudydatarequest.com/Study-Sponsors/Study-Sponsors-Astellas. aspx.

\section{Ethics approval and consent to participate}

The study was conducted in accordance with the protocol, International Council for Harmonization of Technical Requirements for Pharmaceuticals for Human Use $(\mathrm{ICH})$ guidelines, applicable regulations and guidelines governing clinical study conduct, and the ethical principles that have their origin in the Declaration of Helsinki. Written informed consent was obtained from each study patient prior to any study-related procedures. The study protocol and all materials used to obtain informed consent were reviewed and approved by an Institutional Review Board at each study site to ensure that patients' human rights, safety, and well-being were protected.

\section{Consent for publication}

Not applicable.

\section{Competing interests}

T. Takeuchi reports grants from Astellas Pharma, Inc.; Chugai Pharmaceutical Co, Ltd.; Daiichi-Sankyo Co., Ltd.; Takeda Pharmaceutical Co., Ltd.; AbbVie GK; Asahikasei Pharma Corp.; Mitsubishi Tanabe Pharma Co.; Pfizer Japan Inc:; Eisai Co., Ltd.; AYUMI Pharmaceutical Corporation; Nipponkayaku Co. Ltd.; Novartis Pharma K.K.; and Shionogi \& Co., LTD and personal fees from Astellas Pharma, Inc.; AbbVie GK; AYUMI Pharmaceutical Corporation; Eisai Co., Ltd.; Gilead Sciences, Inc.; GlaxoSmithKline K.K.; Sanofi K.K.; Taiho Pharmaceutical Co., Ltd.; Mitsubishi Tanabe Pharma Co.; Daichi Sankyo Co. Ltd.; Chugai Pharmaceutical Co., Ltd.; Taisho Pharmaceutical Co., Ltd.; Eli Lilly Japan K.K.; Novartis Pharma K.K.; Boehringer-Ingelheim Co., Ltd.; Nipponkayaku Co. Ltd.; Pfizer Japan Inc.; Bristol-Myers K.K.; Janssen Pharmaceutical K.K.; and UCB Japan Co. Ltd.

Y. Tanaka reports personal fees from Daiichi-Sankyo; Astellas Pharma, Inc.; Chugai; Eli Lilly; Pfizer; AbbVie; YL Biologics; Bristol-Myers; Takeda; MitsubishiTanabe; Novartis; Eisai; Janssen; and Teijin and grants from Asahi-kasei, Mitsubishi-Tanabe, Chugai, Takeda, Sanofi, Bristol-Myers, UCB, Daiichi-Sankyo, Eisai, Ono.

C. Lademacher, J. Erdman, Y. Kaneko, M. Saito, C. Higashitani, and R. Smulders are employees of Astellas Pharma, Inc.

\section{Author details}

${ }^{1}$ Keio University School of Medicine, 35 Shinanomachi, Shinjuku City, Tokyo 160-8582, Japan. ${ }^{2}$ The First Department of Internal Medicine, University of Occupational and Environmental Health, Kitakyushu, Japan. ${ }^{3}$ Astellas Pharma Global Development, Northbrook, IL, USA. ${ }^{4}$ Astellas Pharma, Inc., Tokyo, Japan.

Received: 28 March 2020 Accepted: 2 October 2020

Published online: 21 October 2020

\section{References}

1. Amaya-Amaya J, et al. Rheumatoid arthritis. In: Autoimmunity: from bench to bedside. Bogota: El Rosario University Press; 2013.

2. Schett $\mathrm{G}$, Gravallese E. Bone erosion in rheumatoid arthritis: mechanisms, diagnosis and treatment. Nat Rev Rheumatol. 2012;8(11):656-64.

3. Scott DL, Wolfe F, Huizinga TW. Rheumatoid arthritis. Lancet. 2010; 376(9746):1094-108. 
4. Haraoui B, et al. Treating rheumatoid arthritis to target: multinational recommendations assessment questionnaire. Ann Rheum Dis. 2011;70(11): 1999-2002.

5. Kameda $\mathrm{H}$, et al. Etanercept (ETN) with methotrexate (MTX) is better than ETN monotherapy in patients with active rheumatoid arthritis despite MTX therapy: a randomized trial. Mod Rheumatol. 2010;20(6):531-8.

6. Kaneko $Y$, et al. Comparison of adding tocilizumab to methotrexate with switching to tocilizumab in patients with rheumatoid arthritis with inadequate response to methotrexate: 52-week results from a prospective, randomised, controlled study (SURPRISE study). Ann Rheum Dis. 2016;75(11): 1917-23.

7. Genovese MC, et al. VX-509 (decernotinib), an oral selective Janus kinase 3 inhibitor, in combination with methotrexate in patients with rheumatoid arthritis. Arthritis Rheumatol. 2015. https://doi.org/10.1002/art.39473 [Epub ahead of print].

8. Singh JA, et al. 2015 American College of Rheumatology Guideline for the treatment of rheumatoid arthritis. Arthritis Rheumatol. 2016;68(1):1-26.

9. Kaneko Y, Takeuchi T. Targeted antibody therapy and relevant novel biomarkers for precision medicine for rheumatoid arthritis. Int Immunol. 2017;29(11):511-7.

10. Takeuchi T. Treatment of rheumatoid arthritis with biological agents - as a typical and common immune-mediated inflammatory disease. Proc Japan Acad Series B. 2017;93(8):600-8.

11. Saeki $Y$, et al. Current treatments of rheumatoid arthritis: from the 'NinJa' registry. Expert Rev Clin Immunol. 2012;8(5):455-65.

12. Kaneko Y, Kondo H, Takeuchi T. American College of Rheumatology/ European League Against Rheumatism remission criteria for rheumatoid arthritis maintain reliable performance when evaluated in 44 joints. J Rheumatol. 2013;40(8):1254-8.

13. SIMPONI ARIA (golimumab) [package insert]. Horsham: Janssen Biotech, Inc; 2017

14. XELJANZ (tofacitinib) [package insert]. Pfizer Inc: New York; 2018.

15. RINVOQ (upadacitinib) [package insert]. Chicago: AbbVie Inc; 2019.

16. ACTEMRA (tocilizumab) [package insert]. South San Francisco: Genentech, Inc; 2019.

17. Tasaki $\mathrm{S}$, et al. Multi-omics monitoring of drug response in rheumatoid arthritis in pursuit of molecular remission. Nat Commun. 2018;9(1):2755.

18. Mor A, Abramson SB, Pillinger $\mathrm{MH}$. The fibroblast-like synovial cell in rheumatoid arthritis: a key player in inflammation and joint destruction. Clin Immunol. 2005;115(2):118-28.

19. Neumann E, et al. Rheumatoid arthritis progression mediated by activated synovial fibroblasts. Trends Mol Med. 2010;16(10):458-68.

20. Noss EH, Brenner MB. The role and therapeutic implications of fibroblast-like synoviocytes in inflammation and cartilage erosion in rheumatoid arthritis. Immunol Rev. 2008:223:252-70.

21. Koch $A E$, et al. Epithelial neutrophil activating peptide-78: a novel chemotactic cytokine for neutrophils in arthritis. J Clin Invest. 1994;94(3): 1012-8.

22. Leech MT, Morand EF. Fibroblasts and synovial immunity. Curr Opin Pharmacol. 2013;13(4):565-9.

23. Croft AP, et al. Distinct fibroblast subsets drive inflammation and damage in arthritis. Nature. 2019;570(7760):246-51.

24. Wynn TA. Two types of fibroblast drive arthritis. Nature. 2019;570(7760): 169-70.

25. Zhang F, et al. Defining inflammatory cell states in rheumatoid arthritis joint synovial tissues by integrating single-cell transcriptomics and mass cytometry. Nat Immunol. 2019;20(7):928-42.

26. Miranti CK, Brugge JS. Sensing the environment: a historical perspective on integrin signal transduction. Nat Cell Biol. 2002;4(4):E83-90.

27. Yokosaki $Y$, et al. The integrin alpha 9 beta 1 mediates cell attachment to a non-RGD site in the third fibronectin type III repeat of tenascin. J Biol Chem 1994;269(43):26691-6.

28. Smith LL, et al. Osteopontin N-terminal domain contains a cryptic adhesive sequence recognized by alpha9beta1 integrin. J Biol Chem. 1996;271(45): 28485-91.

29. Taooka $Y$, et al. The integrin alpha9beta1 mediates adhesion to activated endothelial cells and transendothelial neutrophil migration through interaction with vascular cell adhesion molecule-1. J Cell Biol. 1999;145(2): 413-20

30. Gupta SK, Vlahakis NE. Integrin alpha9beta1: unique signaling pathways reveal diverse biological roles. Cell Adhes Migr. 2010;4(2):194-8.
31. Hoye AM, et al. The newcomer in the integrin family: integrin alpha9 in biology and cancer. Adv Biol Regul. 2012;52(2):326-39.

32. Asano T, et al. Alpha9beta1 integrin acts as a critical intrinsic regulator of human rheumatoid arthritis. Rheumatology (Oxford). 2014;53(3):415-24.

33. Emori $\mathrm{T}$, et al. Constitutive activation of integrin alpha9 augments selfdirected hyperplastic and proinflammatory properties of fibroblast-like synoviocytes of rheumatoid arthritis. J Immunol. 2017;199(10):3427-36.

34. Kanayama $M$, et al. a9 $\beta 1$ integrin-mediated signaling serves as an intrinsic regulator of pathogenic Th17 cell generation. J Immunol. 2011;187(11): 5851-64.

35. Yamamoto $\mathrm{N}$, et al. Essential role of the cryptic epitope SLAYGLR within osteopontin in a murine model of rheumatoid arthritis. J Clin Invest. 2003; 112(2):181-8.

36. Uede T. Osteopontin, intrinsic tissue regulator of intractable inflammatory diseases. Pathol Int. 2011:61(5):265-80.

37. Sugahara S, Hanaoka K, Yamamoto N. Integrin, alpha9 subunit blockade suppresses collagen-induced arthritis with minimal systemic immunomodulation. Eur J Pharmacol. 2018:833:320-7.

38. Huang J, et al. Multiple dose safety, tolerability, pharmacokinetics, and pharmacodynamics of ASP5094, an anti-alpha9 integrin monoclonal antibody, in patients with rheumatoid arthritis on methotrexate [abstract] Arthritis Rheumatol. 2018:70(suppl 10).

39. Tanaka Y, et al. Efficacy and safety of Baricitinib in Japanese patients with active rheumatoid arthritis receiving background methotrexate therapy: a 12-week, double-blind, randomized placebo-controlled study. J Rheumatol. 2016:43(3):504-11.

40. Yamamoto K, et al. Efficacy and safety of certolizumab pegol plus methotrexate in Japanese rheumatoid arthritis patients with an inadequate response to methotrexate: the J-RAPID randomized, placebo-controlled trial. Mod Rheumatol. 2014;24(5):715-24.

41. Tanaka $Y$, et al. Golimumab in combination with methotrexate in Japanese patients with active rheumatoid arthritis: results of the GO-FORTH study. Ann Rheum Dis. 2012;71(6):817-24.

42. Kanayama $M$, et al. Alpha9 integrin and its ligands constitute critical joint microenvironments for development of autoimmune arthritis. J Immunol. 2009;182(12):8015-25.

43. Ohshima S, et al. Enhanced local production of osteopontin in rheumatoid joints. J Rheumatol. 2002;29(10):2061-7.

\section{Publisher's Note}

Springer Nature remains neutral with regard to jurisdictional claims in published maps and institutional affiliations.
Ready to submit your research? Choose BMC and benefit from:

- fast, convenient online submission

- thorough peer review by experienced researchers in your field

- rapid publication on acceptance

- support for research data, including large and complex data types

- gold Open Access which fosters wider collaboration and increased citations

- maximum visibility for your research: over $100 \mathrm{M}$ website views per year

At $\mathrm{BMC}$, research is always in progress.

Learn more biomedcentral.com/submissions 